\title{
A Simple Feed Model that Reduces Time Steps Needed for FDTD Antenna and Microstrip Calculations
}

\author{
R. J. Luebbers, Fellow, IEEE, and H. S. Langdon
}

\begin{abstract}
The finite-difference time-domain (FDTD) method is being widely applied to antenna and microstrip calculations. One aspect of this application is accurately and efficiently modeling antenna and microstrip feeds within the constraints of FDTD approximations. Several relatively straightforward approaches have been suggested, including gap and frill feeds. More complicated approaches, which involve including the coaxial feed cable in the FDTD calculation space, have also been suggested. A related aspect is the desirability of reducing the number of time steps required for FDTD calculations to converge, especially for transient excitation. In this paper we illustrate that for many geometries a simple gap model with an internal source resistance provides accurate results while greatly reducing the number of time steps required for convergence.
\end{abstract}

\section{INTRODUCTION}

$\mathbf{T}$ THE finite-difference time-domain (FDTD) method [1], [2] has become an increasingly popular approach for analyzing the electromagnetic performance of antennas and microstrip devices. With transient excitation, it provides impedance and scattering parameters over a wide frequency band with one calculation and application of the fast Fourier transformation (FFT). One difficulty with FDTD is that for some applications, tens or even hundreds of thousands of time steps may be required for the transient fields to decay.

Previous authors have used different approaches to try to reduce the number of required time steps. In [3], for example, the transient excitation source for a microstrip line is located near the FDTD outer boundary. After the transient source amplitude has fallen to zero, the source is removed and replaced with the FDTD absorbing boundary. While this approach reduced the number of time steps needed, it is awkward to apply for general geometries. It also requires that the feed location be far enough from any geometrical features so that no reflections return to the feed location before the source is removed and the outer absorbing boundary is switched on.

A related approach has been applied to antennas with coaxial cable feeds. Rather than introducing an absorbing boundary during the calculation, a portion of the coaxial cable

Manuscript received June 12, 1995; revised October 20, 1995.

The authors are with the Electrical Engineering Department, The Pennsylvania State University, University Park, PA 16802 USA.

Publisher Item Identifier S 0018-926X(96)04746-1. terminated in an absorbing boundary is included in the FDTD calculation [4], [5] explicitly. The absorbing boundary helps dissipate energy reflected back to the source. In [5] it is claimed that this approach is preferable to the gap source described in [6] since the source fields decay more rapidly with the explicit coaxial feed. Of course, the inclusion of the coaxial line in the FDTD calculation is more cumbersome than the simple gap-feed approach.

A more complicated approach is to apply signal processing methods to predict the voltages and currents at later times from the results computed for early times. Instead of making FDTD calculations for the full number of time steps required for transients to dissipate, one might make actual FDTD calculations for some fraction of this total number of time steps, and use these results to predict those for the later times. A number of papers advocating variations of this approach have appeared. Two recent ones are [7] and [8], and there are a number of earlier attempts cited in these.

Applying the various prediction methods adds additional complexity to the FDTD calculation process. The prediction methods are complicated, and may require care and skill by the user to obtain accurate results. Most of the methods described require the user to determine the order of the prediction process, related to the number of terms of whatever expansion function is used to approximate the FDTD time signal. A poor choice for the order of the prediction model can result in large prediction errors.

In this paper, we present an extremely simple extension to the gap feed described in [6]. This feed reduces the number of FDTD time steps needed for many resonant antenna and microstrip calculations. The approach is based on using a source with an internal resistance to excite the problem. Several previous authors have considered using resistive voltage sources. An internal source resistance was used in [9] for excitation of microstrip patch antennas. Active and passive lumped elements in two-dimensional (2-D) FDTD calculations were discussed in [10]. Methods for including lumped loads in three-dimensional (3-D) FDTD (including nonlinear lumped loads) were described in [11] and again in [2]. The source voltage for a voltage source with a source resistance in parallel with the free space capacitance of the FDTD cell is given in $[12,(7 b)]$. However, in none of these papers was an illustration of the advantages of using an internal source resistance in reducing FDTD calculation time presented. 


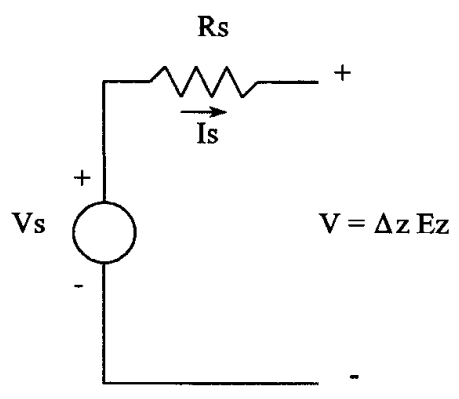

Fig. 1. FDTD source with source resistance $R_{s}$.

In the following sections of this paper, an approach for including the source resistance will be presented and an example calculation for a microstrip antenna will be used to illustrate the resulting reduction in FDTD calculation time.

\section{RESISTIVE SOURCE FDTD EXCITATION}

FDTD antenna or microstrip transient calculations are often excited by a "hard" voltage source such as described in [2], [6]; that is, the internal source resistance is zero ohms. These sources are very easy to implement in an FDTD code. The electric field at the mesh edge where the source is located is determined by some function of time rather than by the FDTD update equations. A common choice is a Gaussian pulse, but other functions may be used. The Gaussian pulse is significantly greater than zero amplitude for only a very short fraction of the total computation time, especially for resonant geometries such as many antennas and microstrip circuits. Once the pulse amplitude drops the source voltage becomes essentially zero, the source effectively becoming a short circuit. Thus, any reflections from the antenna or microstrip circuit which return to the source are totally reflected. The only way the energy introduced into the calculation space can be dissipated is through radiation or by absorption by lossy media or lumped loads. For resonant structures, there are frequencies for which this radiation or absorption process requires a relatively long time to dissipate the excitation energy. Using a source with an internal resistance to excite the FDTD calculation provides an additional loss mechanism for the calculations.

Consider that it is desired to excite an FDTD calculation with a voltage source that corresponds to an electric field $E$ in the $z$ direction at a certain mesh location $i_{s} \Delta x, j_{s} \Delta y, k_{s} \Delta z$, described using the usual Yee notation. The corresponding equivalent circuit for a voltage source which includes an internal source resistance $R_{s}$ is illustrated in Fig. 1. If the source resistance $R_{s}$ is set to zero then the usual FDTD electric field at the source location is simply given by

$$
E_{s}^{n}\left(i_{s}, j_{s}, k_{s}\right)=V_{s}(n \Delta t) / \Delta z .
$$

$V_{s}$ is any function of time--often a Gaussian pulse.

However, with the source resistance included, the calculation of the source field $E_{s}^{n}\left(i_{s}, j_{s}, k_{s}\right)$ at each time step is complicated slightly. To determine the terminal voltage $V$ of Fig. 1 and, thus, the FDTD electric source field $E_{s}^{n}\left(i_{s}, j_{s}, k_{s}\right)$, the current through the source must be determined. This can

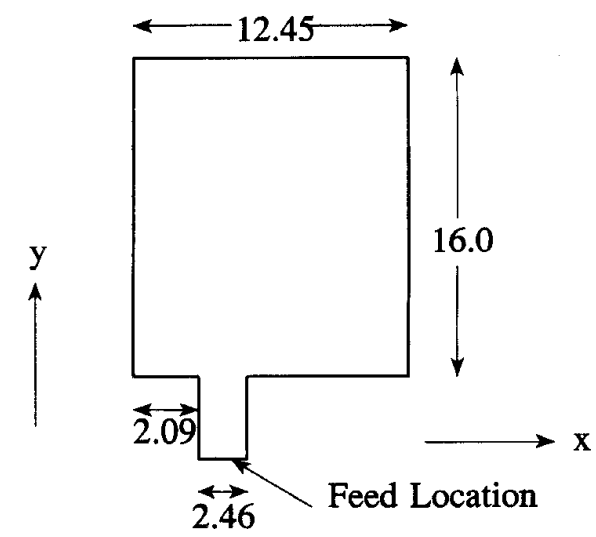

Fig. 2. Geometry of microstrip patch antenna from [3] (all dimensions in $\mathrm{mm})$.

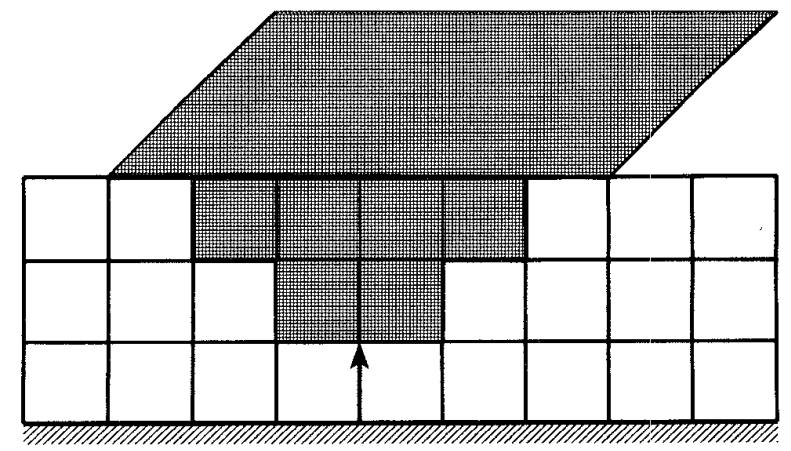

Fig. 3. Detail of staircased FDTD mesh transition from the electric field-source location to the full width of the microstrip feed to the patch antenna.

be done by applying Ampere's circuital law, taking the line integral of magnetic field around the electric field source location. The current through the source is then given by

$$
\begin{aligned}
I_{s}^{n-1 / 2}= & \left(H_{x}^{n-1 / 2}\left(i_{s}, j_{s}-1, k_{s}\right)-H_{x}^{n-1 / 2}\left(i_{s}, j_{s}, k_{s}\right)\right) \Delta x \\
& +\left(H_{y}^{n-1 / 2}\left(i_{s}, j_{s}, k_{s}\right)-H_{y}^{n-1 / 2}\left(i_{s}-1, j_{s}, k_{s}\right)\right) \Delta y
\end{aligned}
$$

so that by applying Ohm's law to the circuit of Fig. 1 the electric source field is given by

$$
E_{s}^{n}\left(i_{s}, j_{s}, k_{s}\right)=V_{s}(n \Delta t) / \Delta z+I_{s}^{n-1 / 2} R_{s} / \Delta z
$$

If $R_{s}=0$ in this equation, then the usual hard-voltage source results.

The 1/2 time-step offset between the current and voltage used in the application of Ohm's law to determine the terminal voltage has not been observed to introduce any appreciable error into the FDTD impedance calculation. As an indication of this, in usual practice, the complex Fourier transforms of the source voltage and current are divided to produce the impedance without any correction needed for the $1 / 2$ time step offset between them. However, the source resistance cannot be made too large or instabilities can occur due to neglecting the displacement current through the FDTD cell containing the 


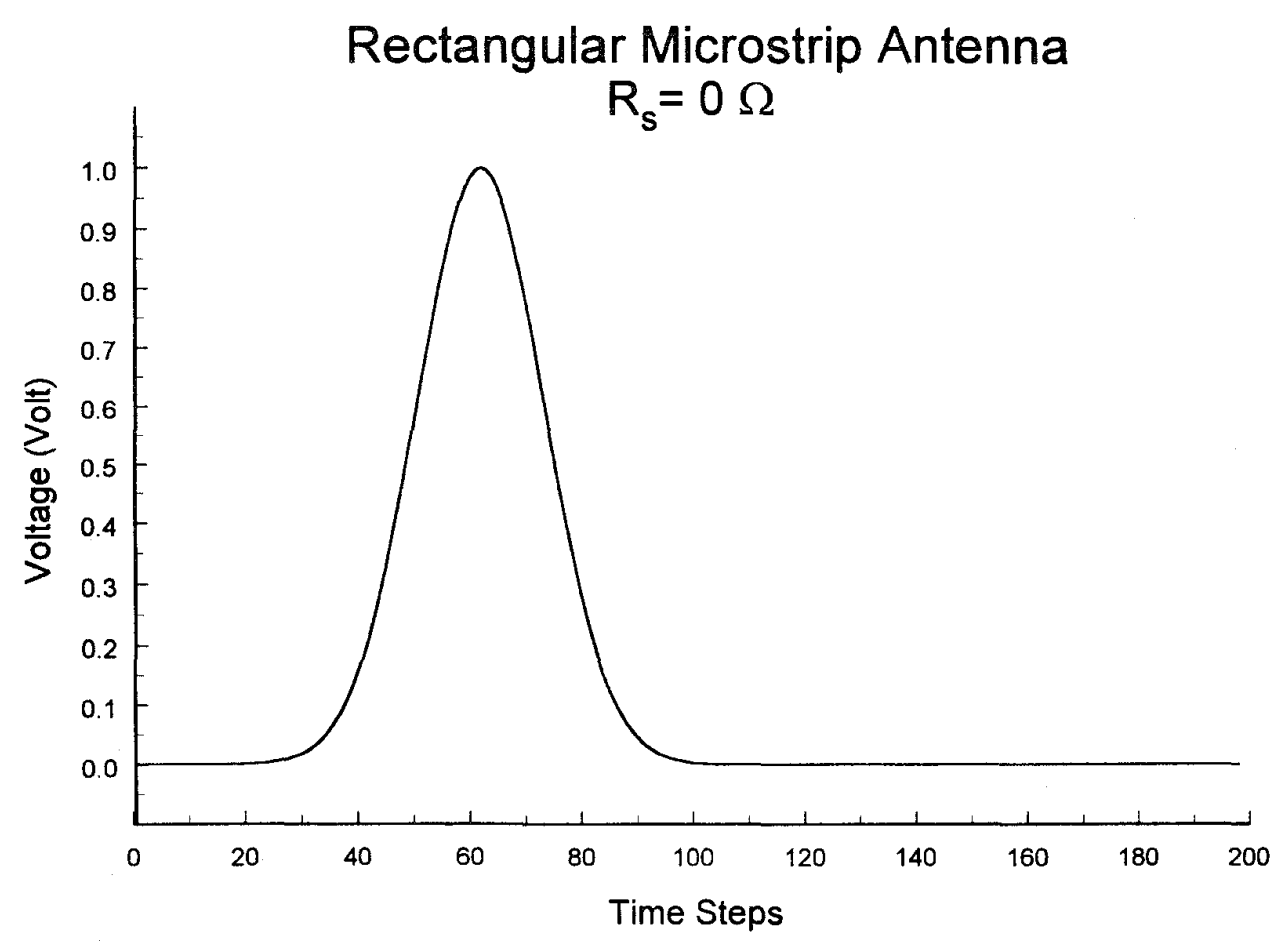

Fig. 4. Patch antenna source voltage $V$ with source resistance $R_{s}=0$. Only the early time steps are shown.

source. If this is a problem, then the source resistance can be reduced, or the capacitance of the FDTD cell can be included as discussed in [11], [2] or as in [12, (7b)].

The value of the internal resistance does not appear to be critical. A reasonable choice for $R_{s}$ is to use the value of the characteristic impedance of the transmission line, coaxial cable, or microstrip, depending on the particular antenna geometry.

\section{EXAMPLE}

To illustrate the advantage of using a source with an internal resistance, $S_{11}$ versus frequency for a rectangular microstrip antenna over a dielectric substrate will be calculated. The geometry is similar to that shown in Fig. 3 of [3]. The only difference is that the $50 \Delta y$ long feed line $(2.46-\mathrm{mm}$ wide in Fig. 2) is reduced to $10 \Delta y$ in the example calculation done here. Thus, the feed location now coincides with the reference plane for phase in [3]. This allows the FDTD space to be smaller, reducing calculation time and memory requirements. The microstrip antenna is located on a $0.794-$ $\mathrm{mm}$ thick dielectric substrate of relative permittivity 2.2 over a ground plane. The cell sizes used are $\Delta x=0.389 \mathrm{~mm}, \Delta y=$ $0.40 \mathrm{~mm}$, and $\Delta z=0.265 \mathrm{~mm}$, the same as in [3]. However the time step used is 0.6407 picoseconds, larger than the 0.441 picosecond time step used in [3]. The problem space size is $69 \times 80 \times 18$ cells. The antenna is fed using a $z$-directed electric field just above the ground plane and directly below the end of the stripline as marked in Fig. 2. This electric fieldsource location is then transitioned to the end of the microstrip feed line by additional FDTD mesh edges of perfect conductor as shown in Fig. 3. In Fig. 3 the perfectly conducting ground plane is at the bottom, the dielectric substrate is shown as unfilled squares, the mesh edge where the electric field feed is located is shown as an arrow, and the conducting meshes are shown filled. The stair-stepped transition from the electric field feed (arrow) to the microstrip line at the top of Fig. 3 was used to provide a relatively smooth connection from the single electric feed location to the microstrip. No comparisons were made with other possible feed geometries. The outer boundary is second order stabilized Liao [13], [14] except for the $z=0$ surface which is the perfectly conducting ground plane.

For the first set of calculations the source resistance $R_{s}$ was set to 0 ohms, which corresponds to the typical FDTD hard source. The source voltage $V$ which determines the FDTD source electric field versus time is shown in Fig. 4. For the hard source with no internal resistance, this is of course just the Gaussian pulse. The corresponding source current $I_{s}$ calculated by FDTD is shown in Fig. 5. This calculation was made for 128000 time steps, and still the current has not completely dissipated.

The corresponding calculations with a source resistance $R_{s}=50$ Ohms are shown in Figs. 6 and 7. The same Gaussian pulse $V_{s}$ was used for both calculations. The source voltage $V$ shown in Fig. 6, determined by saving the source electric field versus time, is no longer just the Gaussian pulse since the voltage across the source resistance $R_{s}$ is also included. The source current $I_{s}$ converges much faster to zero amplitude, reducing the FDTD calculation time by a factor of 32 . Indeed, it appears from Fig. 7 that fewer than 4000 time steps would be sufficient.

What about accuracy? Using the results from both FDTD calculations, shown above, the source voltages and currents 


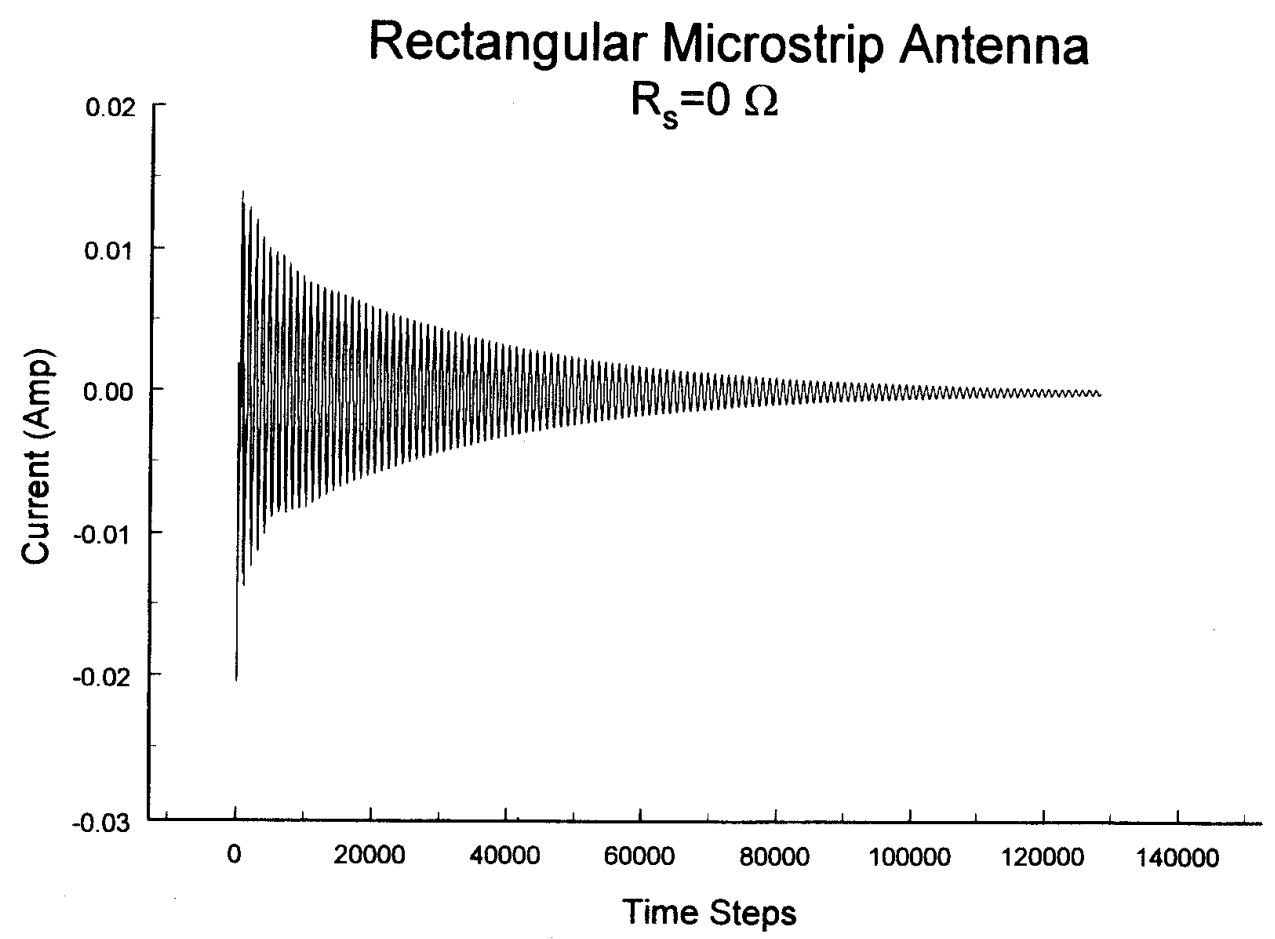

Fig. 5. Patch antenna source current $I_{s}$ with source resistance $R_{s}=0,128,000$ time steps.

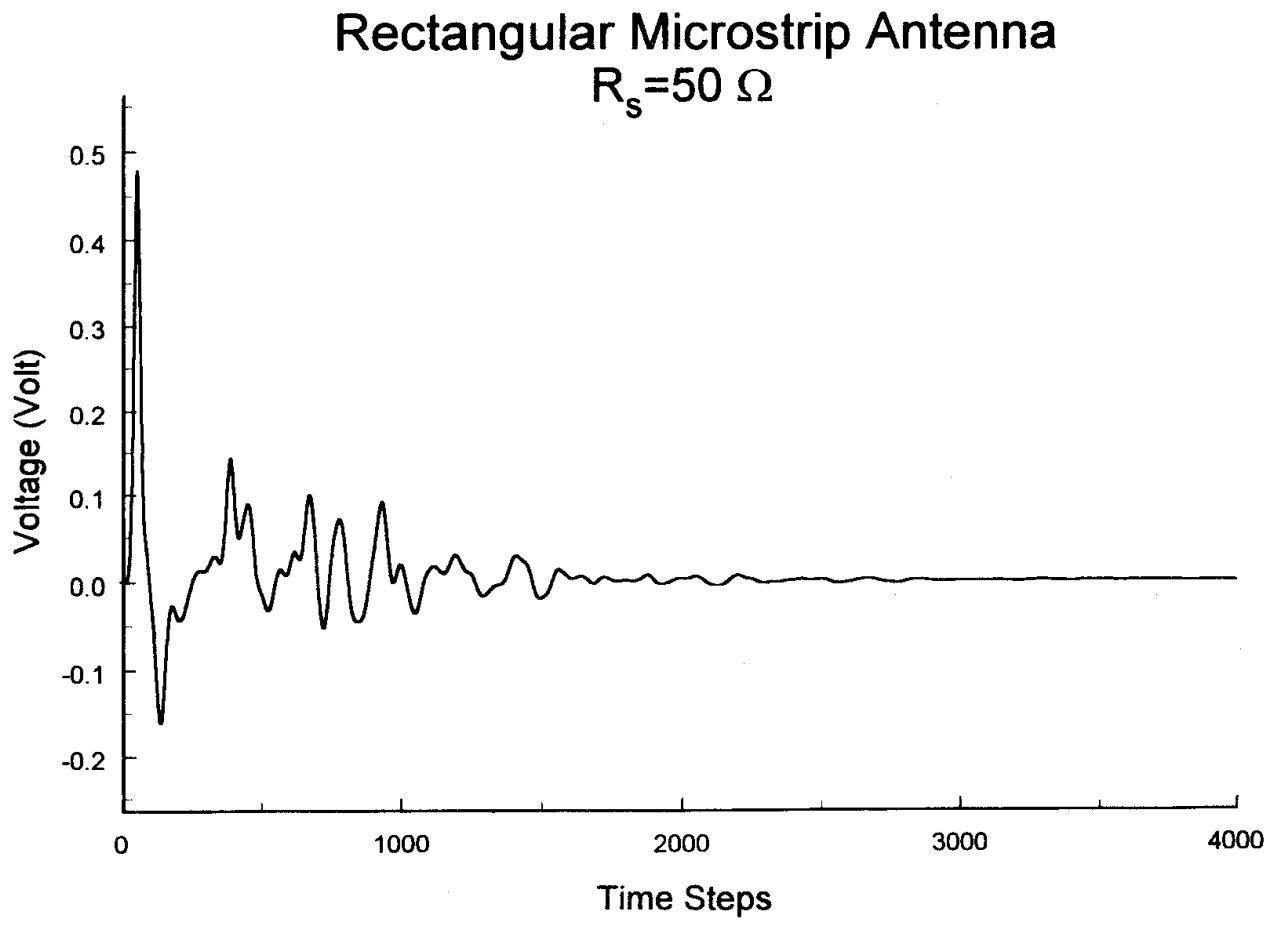

Fig. 6. Patch antenna source voltage $V$ with source resistance $R_{s}=50 \mathrm{ohms}$. All 4000 time steps are shown.

are Fourier transformed using an FFT with the same number of terms. The results for $V$ and $I_{s}$ for 4000 time steps were padded with zeroes to fill the FFT. Then the resulting complex voltages and currents were divided at each frequency to determine the input impedance $Z_{\text {in }}$ at the feed location. The stripline characteristic impedance $Z_{0}$, as in [3], was taken to be 50 Ohms. Then $S_{11}=\left(Z_{\text {in }}-Z_{0}\right) /\left(Z_{\text {in }}+Z_{0}\right)$.

The results obtained for both calculations are shown in Fig. 8. The agreement between the two calculations is excellent, with the 4000 and 128000 time-step curves being 


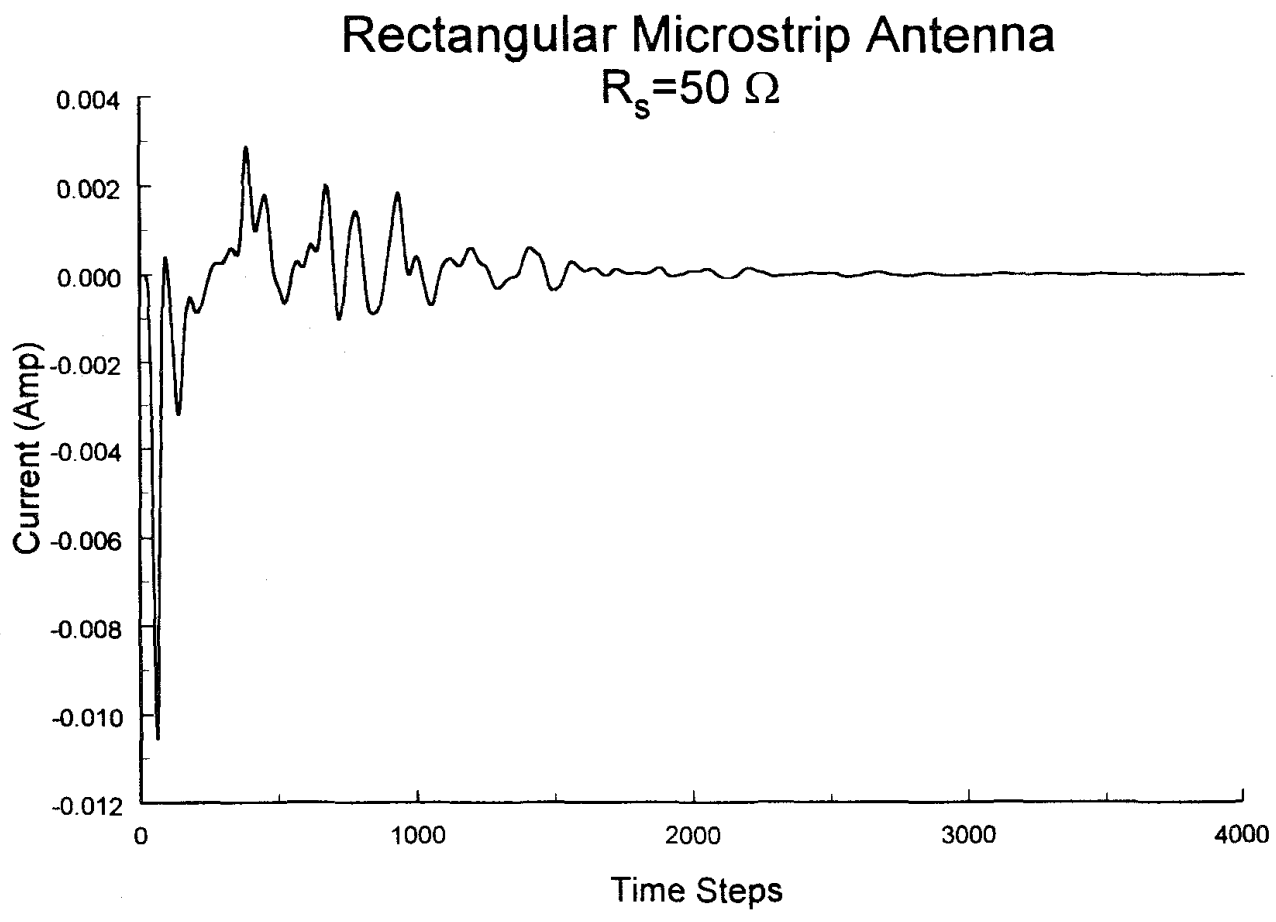

Fig. 7. Patch antenna source current $I_{s}$ with source resistance $R_{s}=50$ Ohms. All 4000 time steps are shown.

Rectangular Microstrip Antenna

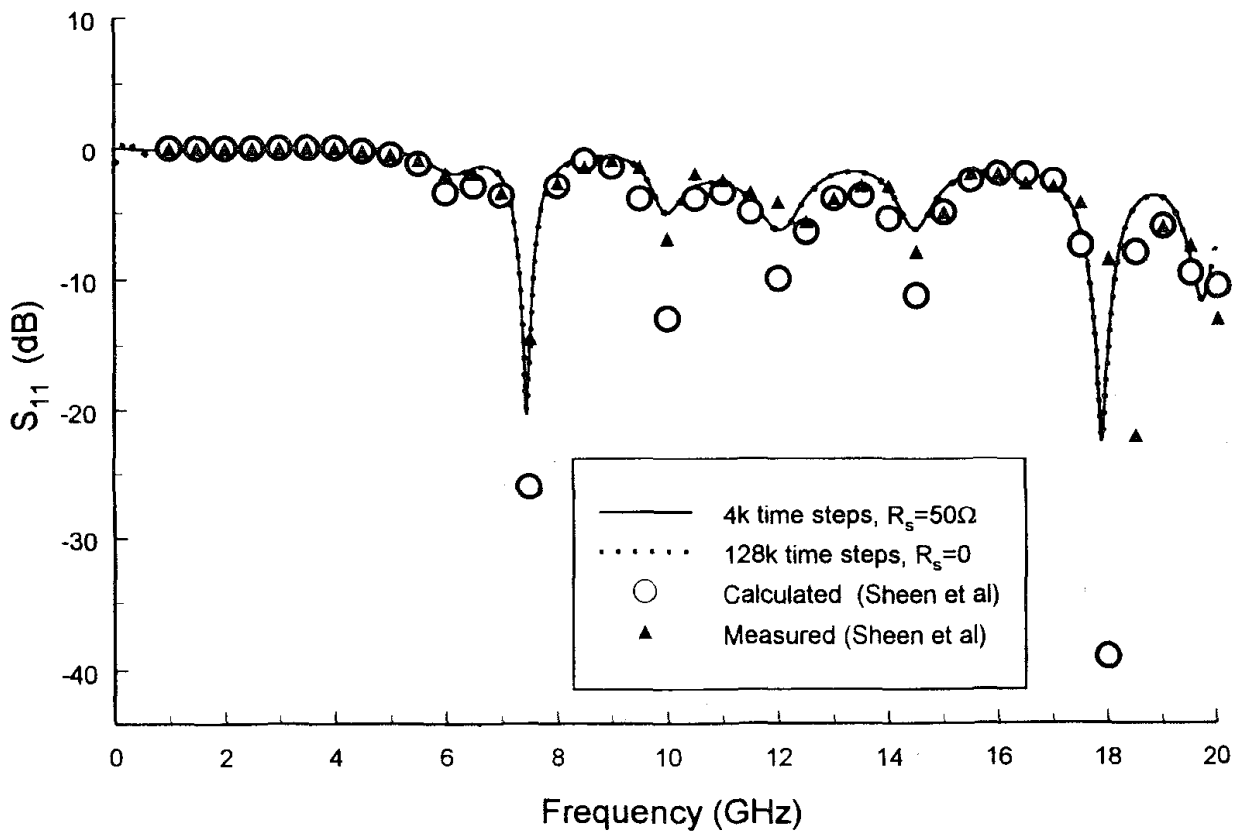

Fig. 8. Return loss parameter $S_{11}$ for both FDTD patch antenna calculations of this paper compared with calculations and measurements from [3].

indistinguishable for most of the plot. The result obtained with $R_{s}=50$ is actually more accurate at the lower frequencies. The result with $R_{s}=0$ still has not quite converged after 128000 time steps, and this causes the ripple in the corresponding low frequency $S_{11}$ results. More importantly, the result with the 50 Ohm source resistance required $1 / 32$ of the computation time of the calculation made with the hard source with $R_{s}=0$. Both results agree well with the measured and calculated results of Sheen et al. [3], which are also shown in Fig. 8. The major discrepancy appears to be in predicting the 
depths of the nulls, with the results obtained in this paper agreeing somewhat better with the measurements than the calculated results given in [3]. Even with the longer stripline feed and removal of the source, the FDTD calculations of [3] required 8000 time steps, twice the number as with the 50 Ohm source resistance.

While very impressive reductions in the number of time steps necessary for convergence have been demonstrated for this geometry, there remain many situations where the signal processing methods such as those described in [7], [8] may be very advantageous. For the source resistance to absorb energy, and thus increase the dissipation rate, the corresponding current must pass through the resistance. This may not happen for more complicated geometries such as two-port microstrip circuits. The number of time steps needed for convergence is not appreciably reduced by including lumped resistances in both ports of the edge-coupled bandpass filter (shown in Fig. 1 of [8]) since current flowing on the central conductor does not flow through either of these resistances. Signal processing methods can be applied to FDTD calculations with plane-wave excitation, while the source resistance approach of this paper cannot.

\section{CONCLUSION}

A simple approach for reducing the number of time steps required for FDTD calculations for resonant antennas and microstrip has been presented. It requires no additional memory, and is simple to implement. No time-varying calculation parameters, complicated post processing, or explicit modeling of a coaxial feed cable is required. For the highly resonant microstrip example shown, accurate results were obtained with $1 / 32$ of the calculation time compared with the FDTD calculation excited by a "hard" voltage source.

\section{REFERENCES}

[1] K. S. Yee, "Numerical solution of initial boundary value problems involving Maxwell's equations in isotropic media," IEEE Trans. Antennas Propagat., vol. AP-14, pp. 302-307, May 1966.

[2] K. Kunz and R. Luebbers, The Finite Difference Time Domain Method for Electromagnetics: Boca Raton, FL: CRC Press, pp. 496, 1993.
[3] D. M. Sheen, S. M. Ali, M. D. Abouzahra, and J. A. Kong, "Application of the three-dimensional finite-difference time-domain method to the analysis of planar microstrip circuits," IEEE Trans. Microwave Theory Tech., vol. 38, pp. 849-857, July 1990 .

[4] J. G. Maloney, G. S. Smith, and W. R. Scott, Jr., "Accurate computation of the radiation from simple antennas using the finite-difference time-domain method," IEEE Trans. Antennas Propagat., vol. 38, pp. 1059-1068, July 1990.

[5] M. Jensen and Y. Rahmat-Samii, "Performance analysis of antennas for hand-held transceivers using FDTD," IEEE Trans. Antennas Propagat., vol. 42, pp. 1106-1113, Aug. 1994.

[6] R. Luebbers, L. Chen, T. Uno, and S. Adachi, "FD'TD calculation of radiation patterns, impedance, and gain for a monopole antenna on a conducting box," IEEE Trans. Antennas Propagat., vol. 40, pp. 1577-1583, Dec. 1992

17] V. Jandhyala, E. Michielssen, and R. Mittra, "FDTD signal extrapolation using the forward-backward autoregressive (AR) model," IEEE Microwave Guide Wave Lett., vol. 4, pp. 163-165, June 1994.

[8] J. Chen, C. Wu, T. K. Y. Lo, Ke-Li Wu, and J. Litva, "Using linear and nonlinear predictors to improve the computational efficiency of the FDTD algorithm," IEEE Trans. Microwave Theory Tech., vol. 42, pp. 1992-1997, Oct. 1994

[9] A. Reineix and B. Jecko, "Analysis of microstrip patch antennas using finite difference time domain method," IEEE Trans. Microwave Theory Tech., vol. 37, pp. 1361-1969, Nov. 1989.

[10] W. Sui, D. A. Christensen, and C. H. Durney, "Extending the twodimensional fdtd method to hybrid electromagnetic systems with active and passive lumped elements," IEEE Trans. Microwave Theory Tech. vol. 40 , pp. $724-730$, Apr. 1992.

[11] R. Luebbers, J. Beggs, and K. Chamberlin, "Finite difference time domain calculation of transients in antennas with nonlinear loads", IEEE Trans. Antennas Propagat, vol. 41, pp. 566-573, May 1993.

[12] M. Piket-May, A. Taflove, and J. Baron, "FDTD modeling of digital signal propagation in 3 - d circuits with passive and active loads, IEEE Trans. Microwave Theory Tech., vol. 42, pp. 1514-1523, Aug. 1994.

[13] Z. P. Liao, H. L. Wong, G. P. Yang, and Y. F. Yuan, "A transmitting boundary for transient wave analysis," Scientia Sinica, vol. 28, no. 10, pp. 1063-1076, Oct. 1984.

[14] M. Moghaddam and W. C. Chew, "Stabilizing liao"s absorbing boundary conditions using single precision arithmetic," Proc. IEEE AP-S Int. Symp., London, ON, Canada, pp. 430-433, 1991.

R. J. Luebbers (S'72-M'76-SM'86-F'95), photograph and biography not available at the time of publication.

H. S. Langdon, photograph and biography not available at the time of publication. 